\title{
Understanding Gender Dysphoria: An Opinion Piece
}

Haley A. Cabe

Augusta University, hcabe@augusta.edu

Follow this and additional works at: https://nsuworks.nova.edu/ijahsp

Part of the Interprofessional Education Commons

\section{Recommended Citation}

Cabe HA. Understanding Gender Dysphoria: An Opinion Piece. The Internet Journal of Allied Health Sciences and Practice. 2017 Jan 01;15(4), Article 3.

This Opinion Piece is brought to you for free and open access by the College of Health Care Sciences at NSUWorks. It has been accepted for inclusion in Internet Journal of Allied Health Sciences and Practice by an authorized editor of NSUWorks. For more information, please contact nsuworks@nova.edu. 


\title{
Understanding Gender Dysphoria: An Opinion Piece
}

\begin{abstract}
This opinion piece on Gender Dysphoria explores the background of the condition, clinical presentation, treatment and prognosis, and specific barriers to care faced by transgender patients. The information provided is based on personal clinical observation as well as scholarly research. There is a clear need for continued exploration into Gender Dysphoria as this is a diagnosis all medical providers may encounter in practice. The purpose of this article is to provide a better understanding of the diagnosis of Gender Dysphoria and to highlight the need for improvement in medical providers' education and clinical skills in order to reduce barriers to care for transgender individuals.
\end{abstract}

\section{Author Bio(s)}

Haley A. Cabe, B.S. RT(T), PA-C, was a student of the class of 2017 Physician Assistant program at Augusta University in Augusta, Georgia. She is also a licensed radiation therapist. 


\title{
1IJAHSP \\ The Internet Joưnal of Allied Health Sciences and Practice
}

Dedicated to allied health professional practice and education

Vol. 15 No. 4 ISSN 1540-580X

\section{Understanding Gender Dysphoria: An Opinion Piece}

\author{
Haley A. Cabe, B.S. RT(T), PA-S \\ Augusta University \\ United States
}

\begin{abstract}
This opinion piece on gender dysphoria explores the background of the condition, clinical presentation, treatment and prognosis, and specific barriers to care faced by transgender patients. The information provided is based on personal clinical observation as well as scholarly research. There is a clear need for continued exploration into gender dysphoria as this is a diagnosis all medical providers may encounter in practice. The purpose of this article is to provide a better understanding of the diagnosis of gender dysphoria and to highlight the need for improvement in medical providers' education and clinical skills in order to reduce barriers to care for transgender individuals.
\end{abstract}

\section{Understanding Gender Dysphoria}

With increased attention in the media, more individuals are coming forward and expressing feelings of gender dysphoria, or gender incongruence. With improved awareness and acceptance, individuals are feeling more comfortable with gender transitioning socially among family and friends. Social transition often includes dressing as the expressed gender as well as using the preferred pronoun or name. The open expression of gender incongruence inspires discussions about the appropriate process of supporting these individuals. The question often arises for parents, family members, and friends: Should we support an individual's desire to transition and if so, to what extent? Medical providers may also question their position on supporting and treating patients with gender dysphoria. In this case, personal opinions should be pushed aside in order to effectively treat patients with compassion and equality.

A study of transgender individuals performed in 2015 including all fifty states of the U.S. reported $60 \%$ of sampled participants were accepted by family members and $68 \%$ were accepted by colleagues. ${ }^{1}$ Another study performed in 2013 revealed $30.3 \%$ of participants reported avoiding medical care due to stigma and marginalization and $15.5 \%$ of the transgender patients sampled were refused medical care at some point in their lifetime. ${ }^{2}$ These studies show an increasing trend of acceptance by family members, coworkers, and friends but a needed improvement in the medical field. This incongruence has been recognized as a problem in recent years with some advancement in standards of care. However, the transgender population remains one of the most underserved groups in medicine.

The first step in improving quality of care for transgender individuals is to understand the diagnosis of gender dysphoria. It is human nature to disregard ideas we do not understand. However, medical providers should strive to be empathetic which requires a basic knowledge of the pathophysiology of the patient's condition. Understanding what a patient with gender dysphoria is experiencing is difficult for some medical providers because unlike atrial fibrillation, for example, there is no test to pinpoint the diagnosis. In the case of gender dysphoria, providers must be attentive to the patient's symptoms and recognize depression and anxiety before a medical emergency occurs.

\section{What is Gender Dysphoria?}

Gender dysphoria is a state of incongruence between one's physical appearance and assigned gender at birth. The development of gender dysphoria diagnosis has evolved since its first recognition in the Diagnostic and Statistical Manual. ${ }^{3}$ Body image is a strong prognostic factor in quality of life, success in relationships, and overall health and wellness. Individuals experiencing gender dysphoria have poor body image and are at increased risk for depression, anxiety, and diminished quality

(C) The Internet Journal of Allied Health Sciences and Practice, 2017 
of life. Thus, the importance of identifying and treating these individuals has become more and more evident. The word identity has a Latin meaning of same. Therefore, gender identity refers to how one relates to sameness compared to others of shared gender. ${ }^{4}$ The gender one identifies with determines the nature of social interactions and one's place in society. In gender dysphoria, the gender one identifies with does not match the sex chromosomes, gonads, and genitalia. This incongruence represents a lack of sameness in relation to others of the shared biological sex and in turn often sparks feelings of distress and dissatisfaction.

Historically, gender dysphoria has been regarded as a phenomenon of psychiatric origin. However, as research has advanced, studies of transgender individuals have revealed minor differences in areas of the brain compared to control groups of cis-gender individuals or individuals who experience sameness in body image and physical characteristics. ${ }^{5}$ These findings have become a compelling reason for further research into the biologic etiology of gender dysphoria and the transgender experience. Currently limited knowledge about the etiology of gender dysphoria contributes to resistance to validation of individuals experiencing it as well as diagnosis and treatment of the condition. However, acceptance of gender differentiation challenges is growing. Increased research will help expand knowledge of how to effectively approach patients with gender dysphoria.

Social transition is often inadequate in addressing anxiety and depression in dysphoric patients. People experiencing gender dysphoria are at higher risk for anxiety and depression. Mental health symptoms are often alleviated with treatment of gender dysphoria using hormone replacement therapy (HRT). HRT involves administration of estrogen or testosterone depending on desired effects. Estrogen replacement in a biological male will produce breast tissue and decrease facial hair. Testosterone replacement in biological females increases facial hair, muscle mass, and lowers voice. Physical changes in the body resulting from HRT often increase feelings of sameness and decrease anxiety and depression.

Mental health evaluation and counseling is a vital component of treatment for gender dysphoric and gender transitioning individuals at the time of diagnosis as well as in preparation for medical transition. Patients undergoing gender transition achieve their greatest outcomes when psychiatric comorbidities remain as low as possible. Effective coordination of care between physicians, advanced practice providers, and mental health professionals is vital when caring for someone considering medical transition. Also, it is important for the patient to understand the direct plan of care in close collaboration with all providers, because best quality of life is achieved by addressing comorbidities simultaneously.

\section{Clinical Presentation of Gender Dysphoria}

Patients experiencing gender dysphoria may present emotionally distraught, anxious, and/or confused. They may report having dysphoric feelings for many years, often beginning in early childhood. ${ }^{6}$ These patients may also describe feelings of inadequacy, reticence in establishing relationships with others, and/or thoughts of self-harm. A study evaluating 104 transgender men and women found these individuals experienced increased difficulty in social interactions and community involvement compared to a control group of non-transgender participants. ${ }^{7}$ This finding is consistent with prior research demonstrating increased prevalence of depression with transgender patients.

Clinicians are likely to discover more information while taking the patient's history as compared to physical exam. Studies show that taking a patient's history provides the most significant findings related to anxiety and depression secondary to gender dysphoria. Until it is explored through interview in a primary care setting, patients may be unable to associate their anxiety and depression to gender incongruence. Therefore, it is important to capture significant historical information due to potentially debilitating depression, anxiety, marginalization, and sometimes even violence experienced by gender dysphoric patients.

Dysphoric feelings are particularly strong during adolescence due to the onset of secondary sexual features. Often, individuals who suppress feelings of gender nonconformity during childhood experience an increase in intensity of dysphoric feelings at the onset of puberty. ${ }^{6}$ Physical findings may be subtle and can include restlessness, eating disorders, or evidence of cutting. When taking the history, it is also important to consider the patient's home-life. If the interaction is with a minor who has a parent present at the time of the visit, a clinician may be able to gauge the parent's ability to recognize mental health symptoms and willingness to support the child's choices. Clinicians may also consider questioning the patient without the parent in order to facilitate an unbiased environment. Candid discussion is an integral feature in determining prognosis for gender dysphoria and medical gender transition.

(C) The Internet Journal of Allied Health Sciences and Practice, 2017 
There is some debate about whether gender dysphoria should be managed by mental health practitioners versus general medical practitioners. Historically, treatment of gender disorders has been addressed only in mental health settings due to lack of understanding of the medical significance of gender incongruence disorders. ${ }^{8}$ Gender dysphoria has not been associated with physical outcomes; therefore, a physical exam was not traditionally included in the evaluation. However, in recent years, there has been a move to a wider range of care for transgender individuals. Important physical findings that might be overlooked are use of devices such as chest binders and prosthetics as well as physical changes seen with HRT and puberty suppression. In conclusion, thorough history and physical exam should be performed when evaluating a patient with gender dysphoria. The diagnosis is determined primarily through history and mental health evaluation, which are more significant than the physical exam. However, the physical exam should not be excluded. Thorough, multi-faceted evaluation should be provided to every patient to ensure quality care no matter the presenting signs and symptoms.

\section{Treatment Options for Gender Dysphoria}

Treating gender dysphoria is imperative to emotional well-being. The anxiety and depression often experienced by transgender patients can be debilitating. After a diagnosis is made, the medical provider should discuss options for treatment which are geared toward resolving dysphoric feelings. As research has evolved, the options for transgender patients have grown. However, barriers to quality medical care still exist. In addition, more complications arise for individuals seeking treatment in childhood or adolescence as compared to adulthood. The two most common forms of medical treatment for gender dysphoria are puberty suppression and HRT. Surgery often follows these treatments.

When considering treatment for children or adolescents, a provider has more factors to consider than when treating adults. One topic of debate is whether the adolescent is mature enough to make a decision that has lifetime effects. Once a thorough and cautious assessment is completed, one option is to suppress puberty at the onset of physical changes by using Gonadotropin-releasing hormone $(\mathrm{GnRH})$ agonists. This treatment prohibits progression of secondary sex characteristics that tend to increase anxiety and depression in patients with gender dysphoria. ${ }^{9} \mathrm{GnRH}$ also provides the patient with time to reach an age where a responsible decision can be made about whether or not to pursue medical transition. One concern is the high cost of puberty suppression therapy. Many families are unable to afford the treatment which then leads to continuation of symptoms of dysphoria into adulthood.

Another alternative for managing transgender patients is HRT, which can be used alone in post-pubertal patients of any age. Prior to initiation of HRT, a baseline evaluation of blood work is obtained and routinely throughout its use. Due to many adverse effects of estrogen and testosterone, this process should be guided by a trained medical professional due to the many adverse effects of estrogen and testosterone. High levels of these hormones may lead to cardiovascular complications especially in those who are at high risk prior to initiation of HRT. Because of barriers to care, many patients turn to illegal purchase and use of hormone replacement drugs. This presents a safety problem due to the nature of HRT and the need for patents to be closely followed by a medical provider.

Surgery is another form of treatment for gender dysphoria. Gender reassignment surgery includes addition or removal of secondary sex organs. According to a study of 55 transgender young adults, patients experience the most improvement in dysphoria after gender reassignment surgery. ${ }^{10}$ The patients in this study had also undergone puberty suppression and HRT. In conclusion, the most effective treatment in relieving anxiety and depression secondary to gender dysphoria is with physical transition using the previously discussed methods. However, it is important to note that insurance companies do not typically cover these treatments, and in turn, patients are presented with financial challenges, another barrier to care.10

\section{Prognosis for Patients with Gender Dysphoria}

Puberty suppression, HRT, and sex-reassignment surgery have all proven to be effective treatments for relieving anxiety and depression in patients experiencing gender dysphoria. Improvement was reported in quality of life $(80 \%)$, psychiatric comorbidities $(78 \%)$, and gender dysphoria (80\%) in a meta-analysis of 28 studies of 1833 transgender individuals who underwent sex-reassignment surgery as well as HRT. ${ }^{11}$ The study also found that individuals with early diagnosis of gender dysphoria had a better prognosis, and those with psychiatric comorbidities tend to have a worse prognosis. The patients who presented earlier were more likely to seek treatment earlier. Those with psychiatric comorbidities were more likely to delay treatment or be inconsistent with therapy. ${ }^{11}$ Suicide and self-harm are currently major public health issues in the transgender population, especially in youth and adolescents. In a systematic review of young people worldwide, the proportion reporting history of suicide attempt was $9.7 \%{ }^{12}$ In comparison, studies in Canada, Europe, and United states report prevalence of suicide attempts in transgender people ranging from 22 to $43 \%$ over a lifetime..$^{13}$

(C) The Internet Journal of Allied Health Sciences and Practice, 2017 
Support from family and friends for one's gender expression and desire to transition plays a large role in treatment prognosis. A study of the mental health of transgender children with a support system resulted in average levels of depression and only minimal elevations in anxiety. The study concludes that psychopathology is not inevitable in the transgender population. ${ }^{14}$ Currently, attention is focused on pre-pubescent children who consistently identify as the opposite sex. With the help of media, acceptance and understanding of gender dysphoria is growing. This equality movement will hopefully continue to spark interest in research and provide individuals, families, and medical providers with accurate and unbiased information about gender dysphoria.

In summary, when evaluating persons with gender dysphoria, it is important for medical professionals to identify mental health symptoms and provide medical advice as early as possible to facilitate early transition if desired, as well as preventing secondary anxiety and depression. Prognosis is greatly improved in cases of early diagnosis of psychiatric comorbidities. The possibility of a positive prognosis creates motivation for medical professionals to improve access to supportive and comprehensive care for individuals experiencing gender dysphoria.

\section{Barriers to Care}

Dysphoric and transgender patients not only have difficulty with access to medical services, transgender patients are often denied basic primary care needs such as pap smears, obstetrics care, or complete denial of all services. Insurance companies sometimes deny routine screening to patients whose gender identity does not coincide with their external genitalia. ${ }^{2}$ Discrimination of transgender patients can be in the form of mandated laws or harassment and occur at all levels of the medical system. Additionally, many transgender patients do not have health insurance, which may be attributed to high unemployment and poverty rates among this population likely related to workplace discrimination. ${ }^{2}$ These examples of barriers to care prevent safe and effective treatment of gender dysphoria and associated anxiety and depression.

Disclosing sexuality with providers presents many difficult obstacles for dysphoric patients. Once patients disclose gender identification to their providers, they may experience judgment and insensitivity depending on the providers' level of knowledge of transgender healthcare. The discrimination experienced by the patient often leads to mistrust of all current and future providers. ${ }^{15}$ Transgender patients often face sexual harassment from practitioners and office staff. Harassment may be in the form of verbal abuse such as being referred to as "It" or inappropriate comments about appearance. Other times, patients are subject to abuse of power by practitioners such as unnecessary physical exams or procedures. A study performed in 2013 reported $24 \%$ of transgender patients experienced verbal or physical harassment in a medical setting. ${ }^{2}$ The need for increased numbers of informed medical professionals who empathize with the needs of gender dysphoria and transgendered patients is paramount.

Because of the lack of knowledge and understanding of gender dysphoria, transgender patients who decide to seek medical treatment are often forced to educate providers on the condition and proper etiquette. Using the patient's preferred pronoun is an important step in effectively caring for a transgender patient. This is difficult for many providers at first, but with diligence, it becomes a natural part of the encounter. Practicing sensitivity when performing the physical exam is especially important with transgender patients. A clinician may encounter a patient wearing a chest binder and should explain to the patient why it may be necessary to remove the chest binder for a thorough heart evaluation. The clinician and patient will feel most comfortable when this interaction is fluid and professional. Overall patient satisfaction is determined not only by quality care but ease and comfort experienced from the provider during the visit.

Another serious barrier to care which dysphoric/transgender patients face is fear of mistreatment that ultimately leads to personal neglect of healthcare. Seeing a healthcare professional can be a stressor for any patient but is especially difficult for transgender patients. The entire process including the waiting room and exam room are opportunities for harassment and selfloathing when a patient is experiencing anxiety or depression related to gender dysphoria. Medical offices can eliminate initial barriers by providing intake forms that include assigned birth sex, gender identity, and preferred name or pronoun. ${ }^{16}$ In addition, providing gender neutral restrooms or not specifying gender on single stall restrooms is another way to decrease anxiety experienced by transgender patients. A recent study of transgender individuals found that $59 \%$ of those surveyed avoided using a public restroom for fear or confrontation or other related problems. ${ }^{1}$ Some individuals may decide their negative expectations of the experience outweigh the benefit of receiving care. This proves the importance for all healthcare personnel to be educated about gender dysphoria and gender transitioning.

When a patient presents with anxiety or depression, providers are often tempted to immediately refer them to psychiatry instead of fully investigating the patient's concerns. This oversight is often a result of the lack of training in the treatment of

(C) The Internet Journal of Allied Health Sciences and Practice, 2017 
mental health symptoms as well as the lack of understanding and knowledge of psychiatric disorders. In addition, there are stigmas associated with disorders of mental health. In the case of anxiety and depression related to gender dysphoria, patients experience many barriers to care directly related to these stigmas. In a study performed in Illinois, 111 transgender people were sampled and $12 \%$ reported being refused routine health care, $3 \%$ were refused mental health care, and $14 \%$ had difficulty with access to emergency care. ${ }^{15}$

Access to proper treatment of gender dysphoria in the United States is most frequently found in urban areas because of increased competence of providers and higher level of acceptance compared to rural areas. These issues present a problem for many transgender Americans such as inconvenience of leaving work early, driving hours for care, and increased travel cost. Another option transgender patients resort to is buying hormone replacement drugs online from other countries. This trend poses an obvious threat given the possibility of unregulated drugs being administered as well as lack of routine lab work essential to HRT maintenance.

\section{Role of Practitioners}

I had the fortunate experience of volunteering with a University-affiliated, student-run clinic serving the lesbian, gay, bisexual, and transgender (LGBT) community. I had the opportunity to surround myself with fellow students and supervising physicians who were well versed on the subjects. One of my most valuable lessons involved an interaction between my attending physician and a new resident who was currently working as an internist. The resident walked in enthusiastically but with reservation about how to treat transgender patients with HRT. She said to the attending "so, how do I do this? I don't see these patients in my practice." The attending politely replied by saying "Actually, you've probably seen several and didn't even know it." Witnessing this interaction impressed me and made me realize how important it is to recognize gender dysphoria as a relevant diagnosis requiring empathy and treatment. This experience, which was optional and not part of mandatory curriculum, has been invaluable to my education and future career. The opportunity allowed me to become familiar with the use of preferred pronouns, learn how to prescribe HRT, and most of all, embed the importance of providing gender affirmative care.

With any medical condition, approaching the patient in an unbiased and objective manner is crucial to quality care. This idea may be easier to implement when dealing with conditions such as hypertension, diabetes, or even cancer. Clinicians encounter primary medical findings frequently and are more prepared for diagnosing, treating, and educating patients with primary medical symptoms. Also, because of the prevalence of these diagnoses, there are fewer stigmas associated. The need to abolish bias and promote objective practice is important in all aspects of medicine and is particularly pertinent when caring for dysphoric or transgender patients. Practicing clinicians also require skills to objectively assess gender identity. This can be a subject that is uncomfortable for both the clinician and patient but one that is crucial to a complete assessment. Clinicians will likely encounter a patient with gender dysphoria at various stages of transition. Some patients will live for many years with anxiety and depression for fear of coming out to family and friends. Others will desire transition on your first interaction. Another group will have already begun or completed transition.

Medical and allied health professionals are often not properly exposed to mental health comorbidities related to gender dysphoria during their respective academic training. This leads to lack of recognition of anxiety, depression, and suicidal ideations in clinical evaluation and treatment of gender dysphoria. Clinicians should educate themselves on signs and symptoms of high-risk patients and be comfortable referring to psychiatry when needed. Many patients will feel some relief from anxiety and depression once the discussion of gender dysphoria is presented. These patients may be treated in the primary care setting alone. Others may have more challenging mental health needs and the primary care practitioner should collaborate care with psychiatry for best clinical outcome.

\section{Conclusion}

Medical providers are often ill equipped to respond effectively to the health needs of patients with gender dysphoria. A survey administered to members of the Society for Adolescent Health and Medicine and the Pediatric Endocrine Society assessed the provider's comfort and confidence in caring for transgender youth aged 8 to 21 . Results showed only $47.1 \%$ felt confident to provide comprehensive care to transgender adolescents and more than $50 \%$ reported lack of training in transgender care. ${ }^{17}$ These results support the need for education and training on transgender care for all primary care providers and especially those working with the pediatric population.

A 2016 survey of endocrinologists showed similar results when dealing with adult transgender patients. Although $80 \%$ of those surveyed reported treating individuals with HRT, $80.6 \%$ reported never receiving training specific to transgender care. ${ }^{18}$ These

(C) The Internet Journal of Allied Health Sciences and Practice, 2017 
results indicate the need for an increase in overall competence among medical providers when caring for the transgender population. A notable point about this survey is the population studied consisted of providers who specialize in HRT and who see a higher percentage of transgender patients. One could conclude the results would be even more discouraging when surveying primary care providers without training who are also likely treating transgender patients.

The need for increased education and training on caring for gender dysphoric and transgender patients is evident and should start with didactic education whether this is medical school or an allied health program. Established clinics and hospitals can develop policies and standards of care specific to transgender health in order to strengthen clinical practice. Organizations such as the National LGBT Health Education Center offer learning modules, webinars, and instructive programs to healthcare organizations requesting support in educating staff on caring for LGBT patients. Resources are available for medical providers who seek improved knowledge, skills, and insight into gender dysphoria and gender transitioning, a growing health matter. Change must start with the individual practitioner; then together, the medical community can make a significant contribution to the lives of many underserved individuals living with the challenges of gender dysphoria and transitioning.

Clinical experience and research broadens awareness on transgender health and the importance of practicing benevolence in every situation. As medical providers, we must always remember the oath to treat all patients equally, with dignity, and to promote the value of diversity. Implementing these principles in our daily practice will not only better the quality of care of the transgender community but of all human beings.

\section{References}

1. James SE, Herman JL, Rankin S, Keisling M, Mottet L, Anafi M. The Report of the 2015 U.S. Transgender Survey. Washington, DC: National Center for Transgender Equality, 2016.

2. Hughto JMW, Murchison GR, Clark K, Pachankis JE, Reisner SL. Geographic and Individual Differences in Healthcare Access for U.S. Transgender Adults: A Multilevel Analysis. LGBT Health. 2016;3(6):424-33. [PMID: 27636030] doi:10.1089/lgbt.2016.0044.

3. Grift TCVD, Cohen-Kettenis PT, Steensma TD, De Cuypere G, Richter-Appelt H, et al. Body Satisfaction and Physical Appearance in Gender Dysphoria. Arch Sex Behav. 2015 Oct;45(3):575-85. [PMID: 26474976]

4. Steensma TD, Kreukels BPC, deVries ALC, Cohen-Kettenis PT. Gender Identity Development in Adolescence. Elsevier Inc.; 2013. Available from: http://www.sciencedirect.com/science/article/pii/s0018506x13000676. Accessed July 10, 2016.

5. Savic I, Arver S. Sex Dimorphism of the Brain in Male-to-Female Transsexuals. Cerebral Cortex. 2011;21(11):2525-33. [PMID: 21467211] doi:10.1093/cercor/bhr032.

6. Forcier M, Kennedy J. Overview of gender development and clinical presentation of gender nonconformity in children and adolescents [Internet]. Overview of gender development and clinical presentation of gender nonconformity in children and adolescents. Available from: http://www.uptodate.com/contents/overview-of-gender-development-and-clinical-presentation-of-gendernonconformity-in-children-and-adolescents?source=see link(4). Accessed July 10, 2016.

7. Davey A, Bouman WP, Meyer C, Arcelus J. Interpersonal Functioning Among Treatment-Seeking Trans Individuals. J Clin Psychol. 2015 Dec;71(12):1173-85. [PMID: 26248509]

8. Wylie K. New standards of care for people with gender dysphoria [Internet]. MHFM; 2008. Available from: http://www.mhfmjournal.com/mental-health/new-standards-of-care-for-people-with-gender-dysphoria.pdf. Accessed July 10, 2016.

9. Tangpricha VI. Transsexualism: Epidemiology, pathophysiology, and diagnosis [Internet]. Transsexualism: Epidemiology, pathophysiology, and diagnosis. Available from: http://www.uptodate.com/contents/transsexualism-epidemiology-pathophysiology-anddiagnosis?source=preview. Accessed July 13, 2016.

10. Shumer DE, Spack NP. Paediatrics: Transgender medicine-long-term outcomes from 'the Dutch model' Nat Rev Urol. 2014 November;12(1):12-3. [PMID: 25403246] doi:10.1038/nrurol.2014.316 
11. Murad MH, Elamin MB, Garcia MZ, Mullan RJ, Murad A, et al. Hormonal therapy and sex reassignment: a systematic review and meta-analysis of quality of life and psychosocial outcomes. Clinical Endocrinology. 2010 Feb;72(2):214-31. [PMID: 19473181]

12. Calear AL, Christensen H, Freeman A, Fenton K, Grant JB, et al. A systematic review of psychosocial suicide prevention interventions for youth. Eur Child Adolesc Psychiatry. 2015 October;25(5):467-82. [PMID: 26472117] doi:10.1007/s00787-015-0783-4

13. Bauer GR, Scheim Al, Pyne J, Travers R, Hammond R. Intervenable factors associated with suicide risk in transgender persons: A respondent driven sampling study in Ontario, Canada. BMC Public Health. 2015 May;15(1):2-13. [PMID: 26032733] doi:10.1186/s12889-015-1867-2

14. Olson KR, Durwood L, Demeules M, Mclaughlin KA. Mental Health of Transgender Children Who Are Supported in Their Identities. Pediatrics. 2016 March;137(3):1-7. [PMID: 26921285]

15. Bradford J, Reisner SL, Honnold JA, Xavier J. Experiences of Transgender-Related Discrimination and Implications for Health: Results From the Virginia Transgender Health Initiative Study. AJPH .2013 October;103(10):1820-9. [PMID: 23153142] doi:10.2105/ajph.2012.300796

16. Reisner SL, Bradford J, Hopwood R, Gonzalez A, Makadon H, Todisco D. Comprehensive Transgender Healthcare: The Gender Affirming Clinical and Public Health Model of Fenway Health. JURH. 2015;92(3):584-92. [PMID: 25779756] doi:10.1007/s11524-015-9947-2.

17. Vance SR, Halpern-Felsher BL, Rosenthal SM. Health Care Providers' Comfort With and Barriers to Care of Transgender Youth. JAH. 2014 November;56(2):251-3. [PMID: 25620310] doi:10.1016/j.jadohealth.2014.11.002

18. Davidge-Pitts C, Nippoldt TB, Danoff A, Radziejewski L, Natt N. Transgender Health in Endocrinology: Current Status of Endocrinology Fellowship Programs and Practicing Clinicians. JCEM. January 2017;2:4. [PMID: 28324050] doi:0.1210/jc.2016-3007. 